\title{
Auguste De la Rive et l'électrothérapie
}

par Isaac Benguigui

\section{RÉsumé}

Après une introduction sur l'état de l'électrothérapie au début du XIX siècle, l'auteur étudie les travaux d'Auguste De la Rive (1801-1873) exposés dans son Traité d'électricité théorique et pratique (1854-1858). Il mentionne les effets thérapeutiques "directs» et "indirects» de l'électricité, les méthodes et les appareils utilisés pour ces effets. Il conclut sur les réserves émises à l'encontre de l'électricité médicale et le peu d'espoir qu'elle suscite encore à la fin du siècle.

Un siècle après la découverte de la bouteille de Leyde ${ }^{1}$ (1745), l'électrothérapie constitue un terrain privilégié pour les travaux des savants du XIX siècle. L'unanimité sur son utilisation et ses méthodes est encore loin d'être acquise. Pour la seule année 1894, on ne compte pas moins de 360 publications sur ce sujet. Les recherches faites par De la Rive en électricité l'amèneront tout naturellement à ses applications médicales.

Auguste De la Rive (1801-1873) appartient à l'une de ces familles qui ont symbolisé la science genevoise au XIX ${ }^{\mathrm{e}}$ siècle. Fils de Gaspard De la Rive, médecin aliéniste et physicien ${ }^{2}$, le nom d'Auguste De la Rive dépassa les frontières de notre pays. Professeur et deux fois recteur à l'Académie, il établit une riche correspondance avec les plus illustres savants d'Europe avec lesquels il se lia d'amitié. Il fit de sa commune de Presinge (Genève) le lieu de rencontre privilégié de nombreux savants européens (Ampère, Arago, Berzélius, Davy, Faraday, auxquels s'ajouteront plus tard les noms de Dumas, Regnault, Verdet, Grove, Sainte-Claire Deville, Helmholtz, Matteucci, Tyndall et d'autres encore).

Membre de plusieurs académies, il dirigea la Bibliothèque Universelle pendant plus d'un quart de siècle. Son œuvre scientifique est considérable. Sa collaboration aux travaux d'Ampère et de Faraday lui donne une dimension européenne ${ }^{3}$.

Quelle est la situation de l'électrothérapie en 1833 au moment où De la Rive publie dans la Bibliothèque Universelle un article intitulé: Esquisse historique sur les principales découvertes faites sur l'électricité depuis quelques années? ${ }^{4}$ 
Dans le Dictionnaire de médecine et de chirurgie pratiques ${ }^{5}$ nous trouvons un article qui nous dit:

«Dans l'état actuel (1831) des connaissances, l'électricité produite par différents appareils peut être introduite dans le domaine de la thérapeutique, non pas comme un moyen spécifique applicable à tous les cas sans distinction, mais comme un agent physique extrêmement puissant, dont les effets peuvent être prévus, calculés, modifiés et dirigés avec plus de facilité et de précision que ne le peuvent être la plupart de médicaments connus... il n'y a que les effets immédiats de l'électricité sur lesquels on puisse compter.»

Dans son Esquisse historique, De la Rive mentionne à peine l'électrothérapie, et encore moins le nom de quelques électrothérapeutes. Il y a là une certaine réserve que nous pouvons comprendre face à l'incertitude qui régnait sur l'état dans lequel était plongé l'électrothérapie. Il faudra attendre une dizaine d'années pour voir se manifester son intérêt pour l'électricité médicale.

Dans le premier numéro des Archives de l'électricité qui est publié comme supplément à la Bibliothèque Universelle, De la Rive nous livre son appréciation :

«C'est dans l'action physiologique de l'électricité qu'on a déjà trouvé et qu'on pourra peutêtre trouver plus tard de puissantes ressources pour l'art médical. L'électricité est un agent dont la médecine devra nécessairement tirer tôt ou tard un parti important. Jusqu'ici, il faut le reconnaître, son emploi n'a pas encore été bien fructueux entre les mains des médecins.»

Ce succès très relatif de l'électricité comme agent thérapeutique est à attribuer, nous dit De la Rive,

«en grande partie, à ce que les physiciens ne sont pas assez physiologistes et à ce que les médecins ne sont pas assez physiciens.» ${ }^{6}$

Quinze ans plus tard, après quelques hésitations, Auguste De la Rive décide de consacrer un chapitre aux applications médicales de l'électricité, intitulé Applications physiologiques, soit thérapeutiques. Ce chapitre est le dernier de son Traité d'électricité théorique et appliquée ${ }^{7}$ publié en trois volumes, entre 1853 et 1858. C'est un ouvrage important qui résume tous les travaux faits dans le domaine de l'électricité. De la Rive donne le point de vue des savants qui en ont fait l'étude et son propre avis. Avec une grande objectivité, il n'hésite pas à confronter les différentes théories ou explications proposées. Il ne s'agit pas d'un ouvrage de vulgarisation. L'auteur nous précise à qui il est destiné «au chimiste aussi bien qu'au physicien, au géologue tout autant qu'au physiologiste, à l'ingénieur comme au médecin». ${ }^{8}$ 
Les réactions à ce traité sont unanimement positives. Faraday qui en a reçu un exemplaire lui écrira:

«Maintenant, quand on me consultera sur un bon Traité d'électricité, je sais ce que je devrai dire. $)^{9}$

Poggendorf attend impatiemment cet ouvrage: «Vos longues et nombreuses expériences dans le domaine de l'électricité ne peuvent qu'exciter au plus haut degré l'attente de tous les physiciens au sujet de la publication de votre traité.» ${ }^{10}$

Léon Foucault, quant à lui, écrira de Paris à De la Rive:

«J'ai eu deux fois l'occasion de parler du Traité d'électricité, ce que je n'ai encore fait pour aucun ouvrage...» ${ }^{11}$

Ce chapitre de l'électricité médicale lui a occasionné plus d'une année de travail. Notons que De la Rive était parfaitement qualifié pour rédiger cet ouvrage. Ses fonctions à l'Académie, ses relations avec les plus illustres de l'Europe savante et ses liens avec un nombre impressionnant de correspondants de plusieurs pays, sa position à la tête de la Bibliothèque Universelle, lui ont certainement facilité la tâche.

Après avoir donné un bref historique de l'électrothérapie, De la Rive nous dit que :

«loin de se généraliser, l'application médicale de l'électricité était tous les jours plus abandonnée, jusqu'au moment où l'on essaya de l'employer sous forme de courant produit par la pile voltaïque». ${ }^{12}$

Pendant le $\mathrm{XIX}^{\mathrm{e}}$ siècle, le galvanisme et le faradisme remplacent progressivement l'électricité statique du siècle précédent. On cherchait à obtenir dans l'emploi de la pile une série de secousses imitant ainsi la série d'étincelles électriques plutôt qu'un courant continu. Les courants d'induction remplissaient mieux que les courants de la pile les conditions stimulantes de l'effet physiologique du courant. A ce propos, mentionnons cette remarque dans le Traité d'électricité médicale à la fin du siècle (1888):

«Les courants continus tombèrent dans une défaveur complète, et ce n'est que dans ces dernières années qu'ils ont de nouveau été employés et qu'ils ont pris pied définitivement dans la thérapeutique médicale.» ${ }^{13}$

Les appareils destinés à fournir l'électricité médicale des courants d'induction se divisent en deux classes ${ }^{14}$ :

a) les appareils volta-électriques dans lesquels l'induction est développée par le courant d'une pile voltaïque (type: Bobine de Ruhmkorff); 
b) les appareils magnéto-électriques dans lesquels l'induction est développée par des aimants permanents (type: machine de Clarke).

\section{Etude des effets thérapeutiques directs de l'électricité}

Par le terme «direct», De la Rive entend une action de l'électricité qui s'excerce physiologiquement et non en vertu de son pouvoir chimique ou calorifique.

Le point de départ de son analyse est que l'électricité est le moyen de communication qui existe entre les centres nerveux et les muscles par l'intermédiaire des nerfs. Il considère (après les travaux de Matteucci et de Dubois-Reymond ${ }^{15}$ qu'il cite souvent) que les muscles et les nerfs ont un état électrique qui leur est propre. Cet état consiste en une disposition particulière des molécules que De la Rive considère comme polarisés. Cet état est maintenu sous l'influence de ce qu'il appelle la force vitale. La propagation de l'action nerveuse s'explique par une modification matérielle dans l'arrangement des particules en vertu de leur polarité électrique.

L'action de l'électricité sur l'organisme s'opère de deux manières: 1) en stimulant les muscles et en remplaçant ou renforçant l'action que les nerfs exercent sur eux, 2) en stimulant les nerfs eux-mêmes, et par conséquent en augmentant ou diminuant l'action naturelle des centres nerveux.

De la Rive insiste sur les propriétés physiologiques et thérapeutiques des deux courants appelés extra-courant et courant induit. Leurs différences sont telles qu' «on ne doit pas employer indifféremment l'un ou l'autre dans la pratique». Les courants induits sont recommandés dans les traitements des atrophies musculaires mais s'avèrent dangereux dans le cas où il s'agit de rendre à des membres paralysés à la suite de paralysie cérébrale leur aptitude motrice.

Dans quels cas faut-il recommander l'emploi de l'électricité? Pour Auguste De la Rive, l'électricité est indiquée pour:

1) rétablir la contractilité dans les muscles qui en sont privés à la suite de lésions encéphalo-rachidiennes;

2) rétablir la sensibilité générale ainsi que la sensibilité spéciale des organes des sens, lorsqu'elles sont abolies ou simplement diminuées;

3) ramener à leur type normal la contractilité et la sensibilité exagérées ou perverties. 
L'électricité est à proscrire, selon De la Rive, dans tous les cas de paralysie provoquée par une lésion du cerveau ou de la moelle.

«Tous les essais qu'on en a faits sur ce rapport ont été parfaitement malheureux et cela devait être.» ${ }^{16}$

\section{Etude des effets thérapeutiques indirects de l'électricité}

De la Rive les définit quand l'électricité n'agit plus en fonction de ses propriétés physiologiques, mais en vertu de ses propriétés chimiques ou physiques. C'est le cas de son emploi pour dissoudre les «calculs de vessie» ou dans le cas où il fut question d'extraire des métaux introduits ou séjournant dans l'organisme, sous forme de remède ou à la suite d'activité professionnelle dans les arts et métiers. Il ne croit pas à l'efficacité de ce traitement. Mentionnons à ce propos que De la Rive fut le lauréat du Prix Montyon décerné par l'Académie des sciences de Paris pour son procédé de la dorure galvanique qu'il substitua à l'ancien procédé au mercure qu'on utilisait dans l'industrie et qui était très nocif pour les ouvriers.

«Frappé des tristes conséquences que représente dans notre ville l'emploi du mercure pour le dorage, j'avais dès longtemps pensé que la force décomposante du courant électrique appliquée à une dissolution d'or, pourrait, en apportant l'or molécule par molécule sur l'objet à dorer, remplacer sinon dans tous les cas, du moins dans plusieurs, l'emploi du mercure...» ${ }^{17}$

Un autre effet thérapeutique indirect de l'électricité est celui de la galvanocaustique, fondé sur les propriétés calorifiques du courant, et qui permet de manier les cautères électriques avec une meilleure précision que celle donnée par la cautérisation au fer rouge.

Quelle est la conclusion concernant tous ces travaux? De la Rive demeure prudent:

«L'électricité peut, quand elle est mal appliquée ou appliquée mal à propos, produire des effets fâcheux... Il en est de ce procédé thérapeutique comme de tous les autres qui, salutaires quand ils sont appliqués à doses modérées, deviennent dangereux quand on les exagère.» ${ }^{18}$

C'est au médecin qu'il fait appel:

«(C'est) à son tact, à son diagnostic et à sa prudence qu'il faut s'en remettre pour la direction à suivre dans l'emploi de cet agent thérapeutique, dont la médecine a déjà tiré parti, mais dont elle a encore, suivant nous, beaucoup à espérer». ${ }^{19}$ 
Cette conclusion, nous pouvons la trouver chez d'autres auteurs qui ont précédé De la Rive de septante à huitante ans. Il s'agit encore de la même prudence dans l'emploi de l'électricité, de la même réserve à son égard, mais aussi du même espoir.

A la fin du siècle, quarante ans après l'ouvrage de De la Rive, chez le public comme chez les praticiens, c'est la prudence qui s'impose. Les médecins sont critiqués pour leur manque de maîtrise dans l'application de l'électricité à la médecine. La physiologie elle-même n'est pas épargnée.

Nous citons quelques conclusions qui nous paraissent traduire l'état d'esprit à la fin du siècle précédent.

Dans son Traité élémentaire et pratique d'électricité médicale, destiné aux médecins, G. Baudet écrit :

«Jusqu'ici malheureusement, l'électricité a été mal connue des médecins, ce qui l'a fait ranger parmi ces agents, actifs à coup sûr, mais mystérieux, dont l'emploi ne peut, en raison même du vague de leurs indications, se faire qu'au hasard et suivant les circonstances.» ${ }^{20}$

Quant à Paul-Louis Ladame, dans un ouvrage publié en 1888, il conclut également sur une note pessimiste:

«Ceux qui ont quelque expérience des applications de l'électricité au traitement des malades, déplorent le peu de lumières que la physiologie, malgré des travaux considérables, a fourni à cette branche de la thérapeutique... Bien loin d'être dirigés par la science physiologique, nous en sommes réduits encore malheureusement presque toujours à l'empirisme le plus complet.» ${ }^{21}$

Il faut le reconnaître, à la fin du siècle l'emploi médical de l'électricité est encore bien limité, et de toutes parts on rencontre, à son égard, des préventions difficiles à dissiper. 


\section{Notes}

1 Cf. notre note, in Théories électriques du XVIII e siècle, Genève, 1984, p. 138.

2 Cf. notre article, Charles-Gaspard De la Rive (1770-1834), médecin aliéniste et physicien, in Gesnerus 42, 1985, p. 245-252.

3 Cf. notre ouvrage Trois physiciens genevois et l'Europe savante : les De la Rive (1800-1920), Genève, 1991.

4 Bibliothèque Universelle 52, 1833, p. 225-264, 404-447, 53, 1833, p. 70-125, 170-227.

5 Andral Gabriel, Dictionnaire de médecine et de chirurgie pratiques, Paris, 1831, t. VII, p. 21, article Electricité.

6 De la Rive Auguste, Coup d'œil sur l'état actuel de nos connaissances en électricité, Archives de l'électricité, avril 1841, p. 25.

7 De la Rive Auguste, Traité d'électricité théorique et appliquée, Paris, 3 vol., 1854-1858. Le premier volume fut publié d'abord en anglais en 1853 et traduit par Charles Vincent Walker. L'édition française parut l'année suivante.

8 De la Rive, loc.cit. (voir note 7), p. VI, (préface).

9 Lettre de Faraday à De la Rive, 21 mars 1856, Papiers De la Rive, Bibliothèque publique et universitaire, Département des manuscrits, Genève, (B.P.U.), Ms 2316, F.F. 85-86.

10 Papiers De la Rive, Ms 2318, F.F. 339-346.

11 Lettre de Léon Foucault à De la Rive, 29 février 1856, Ms 2316, F.F. 182.

12 De la Rive, loc.cit. (voir note 7), p. 565.

13 Onimus Ernest et Legros Charles, Traité d'électricité médicale, recherches physiologiques et cliniques, Paris, 1888, p. 71.

14 Dr. Bonnefoy E., Guide pratique d'électrothérapie, rédigé d'après les travaux et les leçons du Dr. Onimus, Paris, 1882, p. 69.

15 Brazier Mary A.B., Du Bois-Reymond Emil Heinrich (1818-1896), in A History of Neurophysiology in the 19th Century, New York, p. 71-79.

16 Traité d'électricité médicale, vol. III, p.614.

17 Lettre adressée à Arago le 2 avril 1840, in Comptes rendus de l'Académie des sciences de Paris, 1840 , t.X, p. 578.

18 De la Rive, loc.cit. (voir note 7), p. 654 .

19 De la Rive, loc.cit. (voir note 7), p. 655.

20 Dr. Baudet G., Traité élémentaire et pratique d'électricité médicale, Paris, 1884, p. 3.

21 Ladame Paul-Louis, Traitement électrothérapique dans les troubles sexuels chez l'homme, Clermont, 1888, p.I. 


\section{Summary}

\section{Auguste De la Rive and electrotherapy}

After a survey of the state of electrotherapy at the beginning of the 19th century, the author studies the pertinent work of Auguste De la Rive (1801-1873), mainly exposed in the three volumes of his Traité d'électricité théorique et pratique (1854-1858). He mentions «direct» and «indirect» therapeutic effects of electricity as well as methods and apparatus applied and concludes by pointing at the reserved attitude towards the medical use of electricity that still prevailed at the close of the century.

\section{Zusammenfassung}

\section{Auguste De la Rive und die Elektrotherapie}

Nach einleitenden Bemerkungen über den Stand der Elektrotherapie zu Beginn des 19. Jahrhunderts geht der Autor auf die Arbeiten von Auguste De la Rive (1801-1873) ein, die namentlich in seinem dreibändigen Traité d'électricité théorique et pratique (1854-1858) enthalten sind. Er erwähnt die «direkten» und «indirekten» Heilwirkungen der Elektrizität sowie die angewandten Methoden und Apparate und schliesst mit den Bedenken und Zweifeln, die noch am Ende des Jahrhunderts hinsichtlich der medizinischen Anwendung der Elektrizität bestanden.

Dr. Isaac Benguigui

11, Avenue des Cavaliers

CH-1224 Genève 